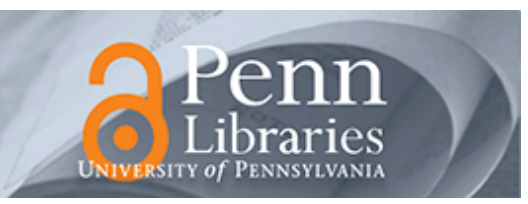

University of Pennsylvania ScholarlyCommons

December 2007

\title{
Relationship between Local Structure and Relaxor Behavior in Perovskite Oxides
}

Ilya Grinberg

University of Pennsylvania

Pavol Juhás

Michigan State University

Peter K. Davies

University of Pennsylvania, davies@lrsm.upenn.edu

Andrew M. Rappe

University of Pennsylvania, rappe@sas.upenn.edu

Follow this and additional works at: https://repository.upenn.edu/mse_papers

\section{Recommended Citation}

Grinberg, I., Juhás, P., Davies, P. K., \& Rappe, A. M. (2007). Relationship between Local Structure and Relaxor Behavior in Perovskite Oxides. Retrieved from https://repository.upenn.edu/mse_papers/141

Copyright 2007 American Institute of Physics. This article may be downloaded for personal use only. Any other use requires prior permission of the author and the American Institute of Physics. Reprinted in Physical Review Letters, Volume 99, Article 267603, December 2007, 4 pages.

Publisher URL: http://dx.doi.org/10.1103/PhysRevLett.99.267603

This paper is posted at ScholarlyCommons. https://repository.upenn.edu/mse_papers/141

For more information, please contact repository@pobox.upenn.edu. 


\title{
Relationship between Local Structure and Relaxor Behavior in Perovskite Oxides
}

\author{
Abstract \\ Despite intensive investigations over the past five decades, the microscopic origins of the fascinating \\ dielectric properties of $\mathrm{ABO}_{3}$ relaxor ferroelectrics are currently poorly understood. Here, we show that \\ the frequency dispersion that is the hallmark of relaxor behavior is quantitatively related to the crystal \\ chemical characteristics of the solid solution. Density functional theory is used in conjunction with \\ experimental determination of cation arrangement to identify the $0 \mathrm{~K}$ structural motifs. These are then \\ used to parametrize a simple phenomenological Landau theory that predicts the universal dependence of \\ frequency dispersion on the solid solution cation arrangement and off-center cation displacements.

\section{Comments} \\ Copyright 2007 American Institute of Physics. This article may be downloaded for personal use only. Any \\ other use requires prior permission of the author and the American Institute of Physics. Reprinted in \\ Physical Review Letters, Volume 99, Article 267603, December 2007, 4 pages. \\ Publisher URL: http://dx.doi.org/10.1103/PhysRevLett.99.267603
}




\title{
Relationship between Local Structure and Relaxor Behavior in Perovskite Oxides
}

\author{
Ilya Grinberg, ${ }^{1}$ Pavol Juhás, ${ }^{2}$ Peter K. Davies, ${ }^{3}$ and Andrew M. Rappe ${ }^{1}$ \\ ${ }^{1}$ The Makineni Theoretical Laboratories, Department of Chemistry, University of Pennsylvania, \\ Philadelphia, Pennsylvania 19104-6323, USA \\ ${ }^{2}$ Department of Physics and Astronomy, Michigan State University, East Lansing, Michigan 48824-2320, USA \\ ${ }^{3}$ Department of Materials Science and Engineering, University of Pennsylvania, Philadelphia, Pennsylvania 19104-6323, USA
}

(Received 11 July 2006; revised manuscript received 3 June 2007; published 27 December 2007)

\begin{abstract}
Despite intensive investigations over the past five decades, the microscopic origins of the fascinating dielectric properties of $A B \mathrm{O}_{3}$ relaxor ferroelectrics are currently poorly understood. Here, we show that the frequency dispersion that is the hallmark of relaxor behavior is quantitatively related to the crystal chemical characteristics of the solid solution. Density functional theory is used in conjunction with experimental determination of cation arrangement to identify the $0 \mathrm{~K}$ structural motifs. These are then used to parametrize a simple phenomenological Landau theory that predicts the universal dependence of frequency dispersion on the solid solution cation arrangement and off-center cation displacements.
\end{abstract}

DOI: $10.1103 /$ PhysRevLett.99.267603

PACS numbers: 77.80.Bh, 77.22. $-\mathrm{d}, 77.84 .-\mathrm{s}$

Since the first synthesis of the classic $\mathrm{PbMg}_{1 / 3} \mathrm{Nb}_{2 / 3} \mathrm{O}_{3}$ (PMN) material in 1961 [1], relaxor ferroelectrics have been the subject of ongoing experimental and theoretical investigation [2-7] due to their fundamental scientific interest and their importance in technological applications such as capacitors and piezoelectric devices [8,9]. One of the most intriguing properties of relaxors is their temperature- and frequency-dependent dielectric response. While normal ferroelectrics exhibit a narrow peak in dielectric constant versus temperature around the ferroelectric phase transition $T_{c}$, relaxors show a broad peak with a full width at half maximum of $50 \mathrm{~K}$ or more (suggesting a "relaxed" transition). Furthermore, the dielectric response and, in particular, the temperature $T_{\epsilon, \max }$ which maximizes the dielectric constant depend strongly on frequency $\omega$. Despite intense research, the relationship between perovskite composition and relaxor properties is poorly understood. While it is known that heterovalency and a degree of disorder on the $B$ site are necessary for relaxor behavior in $\mathrm{Pb}$-based systems, quantitative relationships between local structure and relaxor dispersion are lacking. Here, we show that, taking advantage of the recent advances in the synthesis of Pb-based relaxor perovskites with long-range $B$-cation ordering and the availability of local structural information from density functional theory (DFT) calculations, the extent of relaxor behavior can be predicted from the crystal chemical properties of the constituent ions.

Previously, we explored the properties of the $\mathrm{PbSc}_{2 / 3} \mathrm{~W}_{1 / 3} \mathrm{O}_{3}$ (PSW) perovskite and its solid solution with $\mathrm{PbTiO}_{3}$ (PT) [10,11]. Figure 1(a) compares the dielectric constant frequency dispersion $\Delta T_{\text {disp }}=$ $T_{\epsilon, \max }(100 \mathrm{~Hz})-T_{\epsilon, \max }(1 \mathrm{MHz})$ for PSW-PT with the prototypical PMN-PT solution [12-15]. The magnitude of $\Delta T_{\text {disp }}$ is proportional to the activation barrier $E_{a}$ in the Vogel-Fulcher formula [16,17], often used to characterize relaxor dispersion. The PSW end member exhibits a $40 \%$ larger $\Delta T_{\text {disp }}$ value than PMN. Even more remarkably, the high dispersion of PSW-PT persists for much higher PT concentration than for PMN-PT, with PSW-PT solutions exhibiting $\Delta T_{\text {disp }}$ values larger than that of PMN up to $x_{\mathrm{PT}}=0.35$.

To understand the origins of the unusually large dispersion in PSW-PT solutions, we search for correlations between the experimentally observed $\Delta T_{\text {disp }}$ values and crystal chemical parameters such as ionic valence and ionic displacement. Recent theoretical and experimental research showed that the changes in the local potential energy surface of the $\mathrm{Pb}$ cations are responsible for compositional phase transitions in $\mathrm{Pb}$-based systems [18-20]. $\mathrm{Pb}$ off centering, which gives rise to ferroelectricity in $\mathrm{PbTiO}_{3}$ and derived materials, is due to the energetically favorable formation of short $\mathrm{Pb}-\mathrm{O}$ bonds [21]. For oxygen atoms with high valence $B$-cation neighbors (e.g., two $\mathrm{Nb}$
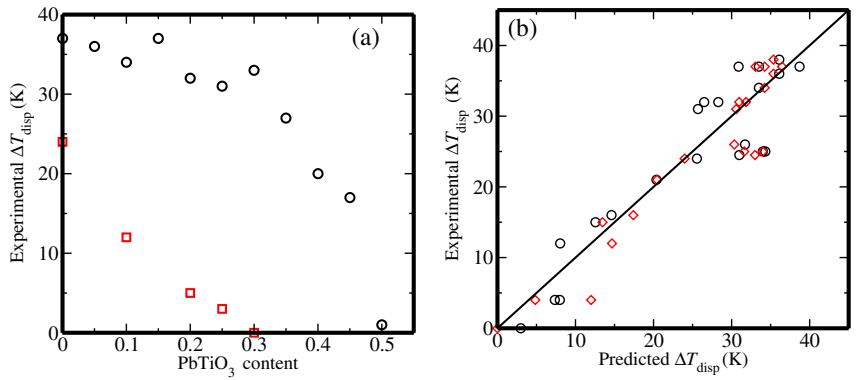

FIG. 1 (color online). (a) Illustration of $\Delta T_{\text {disp }}$ as a quantitative measure of relaxor behavior. $\Delta T_{\text {disp }}$ is plotted as a function of $\mathrm{PbTiO}_{3}$ content in PSW-PT (circles) and PMN-PT (squares) solid solutions. In PSW-PT, dispersion is stronger and persists to much higher PT content. (b) Comparison of local structure model prediction of $\Delta T_{\text {disp }}$ with experimental values for 21 $B$-site ordered relaxor material compositions presented in Table I. Linear model [Eq. (2), circles] and Landau theory model [Eq. (5), diamonds] provide good agreement with experiment yielding average $\Delta T_{\text {disp }}$ errors of 3.7 and $2.9 \mathrm{~K}$, respectively. 
atoms in PMN) such short bonds would lead to oxygen bond order greater than two. This violation of oxygen bond order conservation is unfavorable $[19,22]$. Similarly, oxygen atoms with two low-valence $B$-cation neighbors (e.g., two Sc atoms in PSW) would strongly favor Pb displacement in their direction. This gives rise to variations in the local potential, corresponding to the variation in the value of the $h$ parameter in spin models, which disrupts ferroelectricity and gives rise to the relaxor phase $[23,24]$.

As a measure of the degree of these fluctuations, we use the second moment of the valence of the two $B$-cation nearest neighbors of each oxygen atom $\left(\left\langle V^{2}\right\rangle\right)$, defined as

$$
\left\langle V^{2}\right\rangle=\frac{1}{N_{\mathrm{O}}} \sum_{i}\left(V_{i, 1}^{B}+V_{i, 2}^{B}-2 \bar{V}^{B}\right)^{2},
$$

where the $i$ index runs over all of the $\mathrm{O}$ atoms and $V_{i, 1}$ and $V_{i, 2}$ are the valences of the two nearest $B$-cation neighbors of the $i$ th $\mathrm{O}$ atom. The first moment of the oxygen atom $B$-cation neighbor valence $\bar{V}^{B}$ is equal to four in all $\mathrm{Pb}-$ based perovskites.

The other relevant crystal chemical parameter is the average $B$-cation displacement away from the highsymmetry cubic structure. Our recent research has underscored the importance of cation displacements for compositional phase transitions among the different ferroelectric phases as well as for relaxor-to-ferroelectric transitions $[19,20]$. Larger $B$-cation off-center displacements allow the overbonding (underbonding) of oxygen atoms to be alleviated by motion of the $B$ cations away from (toward) the $\mathrm{O}$ atom, tending to stabilize normal ferroelectric phases relative to relaxor or other disordered phases. The importance of ionic displacements can also be deduced from the sensitivity of relaxor behavior to external pressure. Application of external pressure shortens interatomic distances; in perovskites, pressure also diminishes offcentering displacements [21]. These effects induce relaxor behavior and greatly increase frequency dispersion [25].

To study correlations between local atomic structure and dielectric dispersion, we develop and test our model for all $23 \mathrm{~Pb}$-based relaxor perovskite systems [26-30] (Table I) for which both the local cation order and the dielectric response have been determined experimentally, including all ordered and some disordered systems. These span a variety of compositions, including solid solutions based on the PSW, PMN, $\mathrm{PbSc}_{1 / 2} \mathrm{Nb}_{1 / 2} \mathrm{O}_{3}$ (PSN), and $\mathrm{PbMg}_{1 / 3} \mathrm{Ta}_{2 / 3} \mathrm{O}_{3}$ (PMT) end members as well as $\mathrm{PbCd}_{1 / 12} \mathrm{Mg}_{1 / 4} \mathrm{Nb}_{2 / 3} \mathrm{O}_{3} \quad(\mathrm{PCMN}), \quad \mathrm{PbZn}_{1 / 3} \mathrm{Nb}_{2 / 3} \mathrm{O}_{3}$ $(\mathrm{PZN})$, and $\mathrm{PbSc}_{1 / 2} \mathrm{Ta}_{1 / 2} \mathrm{O}_{3}$ (PST) perovskites. For the ordered systems, $\left\langle V^{2}\right\rangle$ can be computed exactly. For additional systems where full cation order has not been observed, but for which the $\Delta T_{\text {disp }}$ values are constant for a range of $S$ values, $\Delta T_{\text {disp }}$ has been extrapolated to $S=1$ and incorporated in the test set.

To obtain average values of $B$-cation displacements for these systems, we perform DFT calculations using the
TABLE I. Computed $\left\langle V^{2}\right\rangle, D_{B \text {,av }}$, and experimental $\Delta T_{\text {disp }}$ values for solid solutions with random-site ordered $B$-cation arrangement as well as disordered PSN and PST. Also given are $\Delta T_{\text {disp }}$ values predicted by a fit of experimental data to linear function of $\left\langle V^{2}\right\rangle$ and $D_{B \text {,av }}$ [Eq. (2)] and Landau theory predictions based on Eq. (5). References to the experimental $\Delta T_{\text {disp }}$ are given; CS refers to current study and $\mathrm{OM}$ to the references given in the supplementary material [31].

\begin{tabular}{llllccc}
\hline \hline & $\left\langle V^{2}\right\rangle$ & $D_{B \text {,av }}$ & $\Delta T_{\text {disp }}$ & $\Delta T_{\text {disp }}^{\text {lin }}$ & $\Delta T_{\text {disp }}^{\text {Landau }}$ & Reference \\
\hline PSW & 2.0 & 0.10 & 37 & 39 & 37 & {$[10]$} \\
0.95PSW-0.05PT & 1.8 & 0.10 & 36 & 36 & 35 & CS \\
0.90PSW-0.10PT & 1.6 & 0.10 & 34 & 34 & 34 & {$[10]$} \\
0.85PSW-0.15PT & 1.4 & 0.10 & 37 & 31 & 33 & CS \\
0.80PSW-0.20PT & 1.2 & 0.10 & 32 & 28 & 32 & {$[10]$} \\
0.75PSW-0.25PT & 1.0 & 0.10 & 31 & 26 & 31 & {$[10]$} \\
0.70PSW-0.30PT & 1.06 & 0.1 & 32 & 27 & 31 & {$[10]$} \\
0.95PSW-0.05PZ & 1.8 & 0.10 & 38 & 36 & 35 & CS \\
0.90PSW-0.10PZ & 1.6 & 0.10 & 37 & 34 & 34 & {$[10]$} \\
PMN & 2.0 & 0.13 & 24 & 26 & 24 & {$[15]$} \\
0.80PMN-0.20PSN & 1.6 & 0.13 & 21 & 20 & 20 & {$[27]$} \\
0.50PMN-0.50PSN & 1.0 & 0.13 & 15 & 13 & 14 & {$[27]$} \\
0.30PMN-0.70PSN & 0.6 & 0.13 & 4 & 7 & 5 & {$[27]$} \\
PMT & 2.0 & 0.11 & 25 & 34 & 32 & {$[26]$} \\
0.95PMT-0.05PZ & 1.08 & 0.11 & 26 & 32 & 30 & {$[28]$} \\
0.90PMT-0.10PZ & 1.06 & 0.10 & 25 & 34 & 34 & {$[28]$} \\
0.85PMT-0.15PZ & 1.4 & 0.10 & 25 & 31 & 33 & {$[28]$} \\
PCMN & 2.0 & 0.155 & 16 & 15 & 17 & {$[29]$} \\
PZN & 2.0 & 0.17 & 12 & 8 & 15 & {$[30]$} \\
PSN-ord & 0.0 & 0.13 & $0-8$ & 3 & 0 & OM \\
PSN-dis & 2.0 & 0.16 & $5-12$ & 10 & 5 & OM \\
PST-ord & 0.0 & 0.11 & 4 & 8 & 12 & OM \\
PST-dis & 2.0 & 0.14 & 16 & 21 & 21 & OM \\
\hline \hline
\end{tabular}

local density approximation with 40- and 60-atom supercells. For compositions that would require extremely large supercells (e.g., 0.95PSW-0.05PT), we interpolate $D_{B \text {,av }}$ values from the available DFT data for that solid solution. Calculations were performed for 0.75PMT-0.25PZ, PSN, 0.75PMN-0.25PSN, PCMN, and PMT to obtain relaxed structures and DFT $D_{B, \text { av }}$ values for these compositions. Data for PSW-PT, PSW-PZ, PMN-PT, and PZN compositions were taken from previous work $[11,19]$. The calculational details are the same as in previous work [19]. The computed $\left\langle V^{2}\right\rangle, D_{B, \text { av }}$, and the experimental $\Delta T_{\text {disp }}$ values are presented in Table I.

Combining the two crystal chemical parameters, we propose a simple linear relationship between $D_{B, \text { av }},\left\langle V^{2}\right\rangle$, and $\Delta T_{\text {disp }}$, such that

$$
\Delta T_{\text {disp }}=a_{V}\left\langle V^{2}\right\rangle-a_{D} D_{B, \text { av }}+C,
$$

where $a_{V}, a_{D}$, and $C$ are constants. The data in Table I are used to fit the experimentally observed $\Delta T_{\text {disp }}$ to Eq. (2). The $\Delta T_{\text {disp }}$ predicted by the fit $a_{V}, a_{D}$, and $C(13 \mathrm{~K}$, $438 \mathrm{~K} / \AA$, and $56.5 \mathrm{~K}$, respectively) are in good agreement with the observed $\Delta T_{\text {disp }}$ values as shown in Fig. 1(b). 

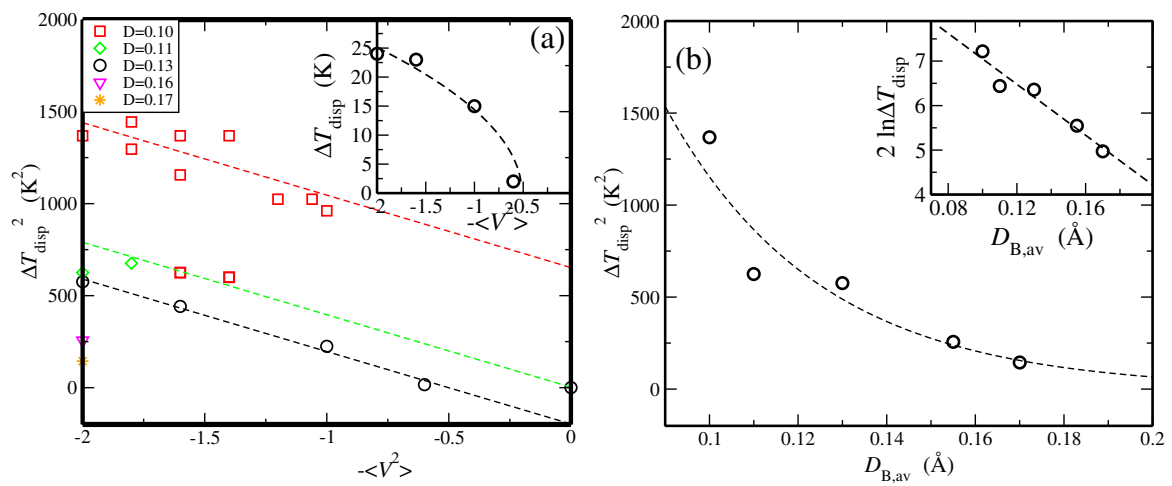

FIG. 2 (color online). (a) Fitting Landau theory model dependence of $\Delta T_{\text {disp }}$ on $-\left\langle V^{2}\right\rangle$ using the data obtained for the PMN-PSN solid solution (inset). Main graph shows linear dependence of $\left(\Delta T_{\text {disp }}\right)^{2}$ on $-\left\langle V^{2}\right\rangle$, with the same slope for all values of $D_{B \text {,av }}$. For $D_{B \text {,av }}=0.10 \AA$, two outliers are seen; these are likely due to slight DFT underestimation of ionic displacements. All data are taken from the $B$-site ordered relaxor composition presented in Table I. (b) Fitting Landau theory model dependence of $\Delta T_{\text {disp }}$ on $D_{B \text {,av }}$, using $B$-site ordered relaxor compositions with $\left\langle V^{2}\right\rangle=2.0$ from Table I. The exponential dependence (main) is confirmed by logarithmic plot (inset).

The connection between crystal chemical properties and dispersion elucidates the differences between PSW-PT and PMN-PT solutions. For the ordered PSW-PT system, weak ferroelectric coupling between $\mathrm{Ti}$ and surrounding $\mathrm{Sc}$ ions suppresses $\mathrm{Ti}$ displacement magnitudes. The $\mathrm{W}$ ions are similarly surrounded by $\mathrm{Sc}$ neighbors and also display small off-center displacements. This makes $D_{B \text {,av }}$ constant (as PT content is increased) and small [11]. Thus, addition of Ti only changes $\left\langle V^{2}\right\rangle$ values, leading to a slow change in frequency dispersion. By contrast, the majority ion in PMN is the ferroelectrically active $\mathrm{Nb}$. This enables $\mathrm{Nb}-\mathrm{Nb}$ displacement coupling even in pure PMN, giving rise to larger $D_{B \text { av }}$ and weaker dispersion than in PSW. As Ti is added into PMN, $\left\langle V^{2}\right\rangle$ decreases and $D_{B \text {,av }}$ increases simultaneously. This leads to rapid suppression of $\Delta T_{\text {disp }}$.

The rather simplistic assumption of linear dependence of $\Delta T_{\text {disp }}$ on $\left\langle V^{2}\right\rangle$ and $D_{B \text {,av }}$ in Eq. (2) is sufficient to demonstrate that a close and quantitative relationship exists between frequency dispersion and crystal chemical parameters. However, careful examination of the data yields a more physically insightful picture. We first focus our discussion on $\mathrm{PbMg}_{1 / 3} \mathrm{Nb}_{2 / 3} \mathrm{O}_{3}-\mathrm{PbSc}_{1 / 2} \mathrm{Nb}_{1 / 2} \mathrm{O}_{3}$ (PMN-PSN) solution data. This system is unique in that it spans the whole range from relaxor PMN to normal ferroelectric PSN, while maintaining long-range random-site cation order [27] and a constant $D_{B \text {,av }}$. These features make it possible to isolate the contribution of $\left\langle V^{2}\right\rangle$ to relaxor dispersion. A plot of $\Delta T_{\text {disp }}$ versus $-\left\langle V^{2}\right\rangle$ shown in the inset of Fig. 2(a) strongly resembles the dependence of the order parameter on temperature in standard Landau theory of a second-order phase transition.

We write the following Landau theory equation to describe the relaxor-to-ferroelectric compositional phase transition

$$
\begin{gathered}
G=G_{0}-\frac{1}{2} A\left(\left\langle V^{2}\right\rangle, D_{B, \text { av }}\right)\left(\Delta T_{\text {disp }}\right)^{2}+\frac{1}{4}\left(\Delta T_{\text {disp }}\right)^{4}, \\
\left(\Delta T_{\text {disp,equil }}\right)^{2}=A\left(\left\langle V^{2}\right\rangle, D_{B, \text { av }}\right),
\end{gathered}
$$

$$
A\left(\left\langle V^{2}\right\rangle, D_{B, \text { av }}\right)=a_{0}+a_{v}\left\langle V^{2}\right\rangle+a_{d} e^{-\kappa D_{B, \mathrm{av}}},
$$

where $G$ is the free energy of the relaxor phase, $G_{0}$ is the energy of the parent ferroelectric phase, and the Landau coefficient $A$ is a function of $\left\langle V^{2}\right\rangle$ and $D_{B, \text { av }}\left(a_{0}, a_{v}, a_{d}\right.$, and $\kappa$ are constants).

The simple linear Curie-Weiss dependence of $A$ on $\left\langle V^{2}\right\rangle$ is extracted from the inset of Fig. 2(a). A plot of $\left(\Delta T_{\text {disp }}\right)^{2}$ versus $\left\langle V^{2}\right\rangle$ [Fig. 2(a)] shows that the data $D_{B \text {,av }}=0.10 \AA$ exhibit the same slope as the $D_{B, \text { av }}=0.13 \AA$ data obtained from the PMN-PSN solid solution. This suggests that the $a_{v}$ coefficient is independent of $D_{B, \text { av }}$ for the $\mathrm{Pb}$-based perovskite solid solutions studied here.

Comparison of frequency dispersion values from perovskites with $\left\langle V^{2}\right\rangle=2.0$ (Table I) shows that $\left(\Delta T_{\text {disp }}\right)^{2}$ exhibits an exponential dependence on $D_{B \text {,av }}$ [Fig. 2(b)]. Such exponential relationship is due to exponential variation of bonding overlap with interatomic distances changed by the cation displacement. The correlation between the experimentally observed $\Delta T_{\text {disp }}$ values and $\Delta T_{\text {disp }}$ values calculated using $a_{0}, a_{v}, a_{d}$, and $\kappa$ values obtained from the fits in Figs. 2(a) and 2(b) is shown in Fig. 1(b). The more physical nature of the modeling gives rise to smaller deviations from experimental results than for the corresponding predictions using the simple linear form in Eq. (2). This indicates that the Landau theory of the relaxor-ferroelectric transition encapsulated by Eq. (5) provides a good model of experimental data. Since the crystal chemical parameters in the model involve individual interatomic interactions, our work strongly suggests that some local, $\AA$-scale interatomic interactions cause relaxor behavior. This supports previously suggested models of relaxors as ferroelectrics with local frustration of the ferroelectric coupling by antiferroelectric interactions [23,32].

For an additional test of our theory, we compare the dispersion predicted for ordered and disordered PSN and PST materials, which have long been used to study the 
effects of $B$-cation ordering on the relaxor-ferroelectric transition (see supplementary material [31]). Using Eq. (5), we obtain $\Delta T_{\text {disp }}$ values of 0 and $15 \mathrm{~K}$ for ordered and disordered PSN, respectively, in agreement with experimentally observed $\Delta T_{\text {disp }}$ values of $0-8$ and $8-12 \mathrm{~K}$ for annealed and as-sintered PSN samples, respectively [3337]. For PST, using Eq. (5) we obtain $\Delta T_{\text {disp }}$ values of 12 and $21 \mathrm{~K}$ for ordered and disordered PSN, respectively. Experimentally, $\Delta T_{\text {disp }}$ is $4 \mathrm{~K}$ for the annealed PST sample and $16 \mathrm{~K}$ for the as-sintered PST sample [38]. The overestimation of the dispersion for ordered PST may be due to the uncertainties in $\left\langle V^{2}\right\rangle$ and $D_{B \text {,av }}(\approx 0.2$ and $\approx 0.01 \AA$, respectively), which are important for systems close to the relaxor-to-ferroelectric phase transition, and may contribute to the overestimation of $\Delta T_{\text {disp }}$ [39].

In conclusion, we have shown that the relaxor or ferroelectric behavior of lead perovskite systems can be predicted by simple Landau theory, where the crystal chemical parameters $D_{B \text {,av }}$ and $\left\langle V^{2}\right\rangle$ change the magnitude of the second-order Landau coefficient. More importantly, the extent of the dielectric dispersion and the strength of the relaxor phase can be quantitatively predicted as well. The exact details of dielectric response in relaxors are still not understood, but we hope that our elucidation of the connections between the structure and the dynamic properties of relaxor materials will stimulate new research to resolve this long-standing problem in condensed matter physics.

This work was supported by the Office of Naval Research, under Grants No. N00014-00-1-0372 and No. N00014-01-1-0860, and by the National Science Foundation, through the MRSEC program, Grant No. DMR05-20020. Computational support was provided by the Center for Piezoelectrics by Design, the DoD HPCMO, DURIP, and by the NSF CRIF program, Grant No. CHE-0131132. We would also like to thank I.-W. Chen and A. A. Bokov for stimulating discussions.

[1] G. A. Smolenskii, V. A. Isupov, A. I. Agranovskaya, and S. N. Popov, Sov. Phys. Solid State 2, 2584 (1961).

[2] I. K. Jeong, T. W. Darling, J. K. Lee, T. Proffen, R. H. Heffner, J.S. Park, K.S. Hong, W. Dmowski, and T. Egami, Phys. Rev. Lett. 94, 147602 (2005).

[3] R. Blinc, J. Dolinsek, A. Gregorovic, B. Zalar, C. Filipic, Z. Kutnjak, A. Levstik, and R. Pirc, Phys. Rev. Lett. 83, 424 (1999).

[4] R. Blinc, V. Laguta, and B. Zalar, Phys. Rev. Lett. 91, 247601 (2003).

[5] V. Westphal, W. Kleeman, and M. D. Glinchuk, Phys. Rev. Lett. 68, 847 (1992).

[6] P. M. Gehring, S. E. Park, and G. Shirane, Phys. Rev. Lett. 84, 5216 (2000).

[7] J. Hemberger, P. Lunkenheimer, R. Fichtl, H. von Nidda, V. Tsurkan, and A. Loidl, Nature (London) 434, 364 (2005).

[8] B.-G. Kim, S. M. Cho, T.-Y. Kim, and H. M. Jang, Phys. Rev. Lett. 86, 3404 (2001).
[9] S.-E. Park and T. R. Shrout, J. Appl. Phys. 82, 1804 (1997).

[10] P. Juhas, M. A. Akbas, and P. K. Davies, J. Am. Ceram. Soc. 87, 2086 (2004).

[11] P. Juhas, I. Grinberg, A. M. Rappe, W. Dmowski, T. Egami, and P. K. Davies, Phys. Rev. B 69, 214101 (2004).

[12] A. A. Bokov and Z. G. Ye, Appl. Phys. Lett. 77, 1888 (2000).

[13] H. X. Wang, H. G. Xu, H. S. Luo, Z. W. Yin, A. A. Bokov, and Z. G. Ye, Appl. Phys. Lett. 87, 021904 (2005).

[14] A. Kania, A. Slodczyk, and Z. Ujma, J. Cryst. Growth 289 , 134 (2006).

[15] A. A. Bokov and Z.-G. Ye, J. Mater. Sci. 41, 31 (2006).

[16] H. Vogel, Phys. Z. 22, 645 (1921).

[17] G. S. Fulcher, J. Am. Ceram. Soc. 8, 339 (1925).

[18] I. Grinberg, V.R. Cooper, and A.M. Rappe, Nature (London) 419, 909 (2002).

[19] I. Grinberg and A. M. Rappe, Phys. Rev. B 70, 220101 (2004).

[20] I. Grinberg, M. R. Suchomel, P. K. Davies, and A. M. Rappe, J. Appl. Phys. 98, 094111 (2005).

[21] R. E. Cohen, Nature (London) 358, 136 (1992).

[22] B. P. Burton and E. Cockayne, Phys. Rev. B 60, R12 542 (1999).

[23] R. Fisch, Phys. Rev. B 67, 094110 (2003).

[24] V. Bobnar, Z. Kutnjak, R. Pirc, R. Blinc, and A. Levstik, Phys. Rev. Lett. 84, 5892 (2000).

[25] G. A. Samara, Phys. Rev. Lett. 77, 314 (1996).

[26] M. A. Akbas and P. K. Davies, J. Am. Ceram. Soc. 80, 2933 (1997).

[27] L. Farber and P. K. Davies, J. Am. Ceram. Soc. 86, 1861 (2003).

[28] M. A. Akbas and P. K. Davies, Int. J. Inorg. Mater. 3, 123 (2001).

[29] A. Kumar, K. Prasad, S. N. Choudhary, and R. N.P. Choudhary, Mater. Lett. 58, 3395 (2004).

[30] Y.-H. Bing, A. A. Bokov, Z.-G. Ye, B. Noheda, and G. Shirane, J. Phys. Condens. Matter 17, 2493 (2005).

[31] See EPAPS Document No. E-PRLTAO-99-032750 for a discussion of the relaxor compositions used in this study, the methodology for the computation of $\left\langle V^{2}\right\rangle$ for perovskites with ordered and disordered $B$-cation arrangement, and a survey of experimental and theoretical results for PST and PSN systems. For more information on EPAPS, see http://www.aip.org/pubservs/epaps.html.

[32] I.-W. Chen, J. Phys. Chem. Solids 61, 197 (2000).

[33] C. Malibert, B. Dkhil, J. M. Kiat, D. Durand, J. F. Berar, and A.S. de Bire, J. Phys. Condens. Matter 9, 7485 (1997).

[34] C. Perrin, N. Menguy, O. Bidault, C. Y. Zahra, A.-M. Zahra, C. Caranoni, B. Hilczer, and A. Stepanov, J. Phys. Condens. Matter 13, 10231 (2001).

[35] H. M. Jang and S.-C. Kim, J. Mater. Res. 12, 2117 (1997).

[36] E. L. Venturini, R. K. Grubbs, G. A. Samara, Y. Bing, and Z.-G. Ye, Phys. Rev. B 74, 064108 (2006).

[37] M. Szafranski, A. Hilczer, and W. Nawrocik, J. Phys. Condens. Matter 16, 7025 (2004).

[38] N. Setter and L. E. Cross, J. Appl. Phys. 51, 4356 (1980).

[39] Despite a high order parameter $S=0.8$, full ordering is not achieved for this system. This introduces uncertainties in $\left\langle V^{2}\right\rangle$ and $D_{B \text {,av }}$. 